\title{
Metal-Metal and Metal-Ligand Bonding in Trinuclear Vinylidene Rhenium-Iron-Platinum Complex Studied by Topological Analysis of Electron Density
}

\author{
Aleksey M. Shora, Svetlana S. Laletina ${ }^{a}$, \\ Anatoly I. Rubaylo ${ }^{\mathrm{a}, \mathrm{b}}$, Elena A. Ivanova-Shor*a \\ ${ }^{a}$ Institute of Chemistry and Chemical Technology SB RAS \\ FRC "Krasnoyarsk Science Center SB RAS" \\ Krasnoyarsk, Russian Federation \\ ${ }^{b}$ Siberian Federal University \\ Krasnoyarsk, Russian Federation
}

Received 10.10.2020, received in revised form 11.11.2020, accepted 19.11.2020

Abstract. Metal-metal and metal-ligand bonding in vinylidene ReFePt complex was studied by density functional method and topological analysis of electron density. Topological analysis did not found direct bonding between the metal atoms pointing to indirect metal-metal interaction mediated via the bridging vinylidene ligand. At the same time, the delocalization index $\delta(\mathrm{Fe}, \mathrm{Pt})$ reveals the strong $\mathrm{Fe}-\mathrm{Pt}$ interaction that allows for supposing a chemical bonding between these atoms.

Keywords: Density functional method, topological analysis, vinylidene complexes, rhenium, platinum, iron.

Citation: Shor A.M., Laletina S.S., Rubaylo A.I., Ivanova-Shor E.A. Metal-metal and metal-ligand bonding in trinuclear vinylidene renium-iron-platinum complex studied by topological analysis of electron density, J. Sib. Fed. Univ. Chem., 2020, 13(4), 553-564. DOI: 10.17516/1998-2836-0205

(c) Siberian Federal University. All rights reserved

This work is licensed under a Creative Commons Attribution-NonCommercial 4.0 International License (CC BY-NC 4.0)

* Corresponding author E-mail address: shor-elena@rambler.ru 


\title{
Изучение взаимодействий металл-металл
}

\author{
и металл-лиганд в трехъядерном винилиденовом \\ рений-железо-платиновом комплексе
}

с помощью топологического анализа

электронной плотности

\author{
А.М. Шор ${ }^{a}$, С.С. Лалетина ${ }^{a}$, \\ А.И. Рубайло, \\ ${ }^{a}$ Институт химии и химической технологии \\ ФИЦ «Красноярский научный центр СО РАН» \\ Российская Федерация, Красноярск \\ ${ }^{6}$ Сибирский федеральный университет \\ Российская Федерация, Красноярск
}

Аннотация. С использованием метода функционала плотности и топологического анализа электронной плотности изучены характеристики взаимодействий металл-металл и металллиганд в винилиденовом комплексе с остовом ReFePt. Топологический анализ указывает на непрямой характер взаимодействия между металлами, осуществляемого посредством мостикового винилиденового лиганда. В то же время индекс делокализации $\delta(\mathrm{Fe}, \mathrm{Pt})$ позволяет предположить существование химической связи между атомами Fe и Pt.

Ключевые слова: метод функционала плотности, топологический анализ, винилиденовые комплексы, рений, платина, железо.

Цитирование: Шор, А.М. Изучение взаимодействий металл-металл и металл-лиганд в трехъядерном винилиденовом рений-железо-платиновом комплексе с помощью топологического анализа электронной плотности / А.М. Шор, С.С. Лалетина, А.И. Рубайло, Е.А. Иванова-Шор // Журн. Сиб. федер. ун-та. Химия, 2020. 13(4). С. $553-$ 564. DOI: $10.17516 / 1998-2836-0205$

\section{Введение}

Синтез гетерометаллических комплексов переходных металлов, содержащих мостиковые винилиденовые лиганды $\mu_{\mathrm{n}} \mathrm{C}=\mathrm{CR}^{\prime} \mathrm{R}^{\prime \prime}\left(\mathrm{n}=2-4, \mathrm{R}^{\prime}\right.$ и $\mathrm{R}^{\prime \prime}=\mathrm{H}$, алкильные и арильные группы и т.п.), получил широкое распространение в последние десятилетия [1-3]. Большинство таких соединений детально охарактеризовано рентгеновскими структурными и различными спектральными (прежде всего ИК и ЯМР) методами. Но существует значительный пробел в изучении данных систем теоретическими методами квантовой химии. Как результат, о характере взаимодействия металл-металл и металл-лиганд в комплексах подобного типа судят в основном исходя из структурных особенностей, опираясь на правило 18 электронов [4].

Как заполнение данного пробела, в текущей работе мы применяем теоретический подход к изучению механизма связывания в трехъядерных ММ>M» $\mu_{3}$-винилиденовых комплексах. В качестве объекта исследования рассматривается комплекс $\mathrm{CpReFePt}\left(\mu_{3} \mathrm{C}=\mathrm{CHPh}\right)(\mathrm{CO})_{6}\left(\mathrm{PPh}_{3}\right)(\mathbf{1})$, 


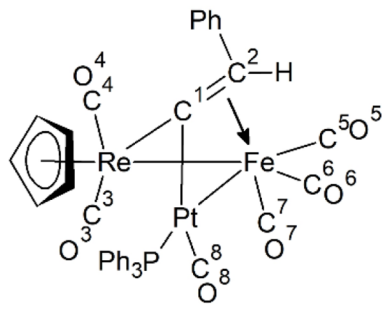

A

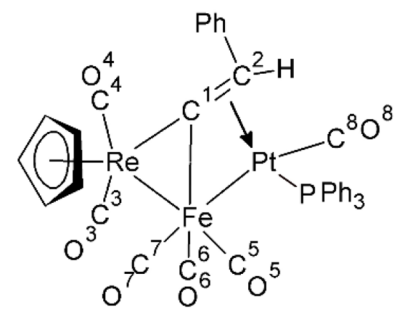

Б

Рис. 1

Fig. 1

который имеет две изомерные формы, отличающиеся типом $\eta^{2}$-координации винилиденового $\mathrm{C}=\mathrm{CHPh}$ лиганда - к атому Fe (A) или к атому Pt (Б) ( $\eta^{2}$-координация показана стрелкой на рис. 1). Отметим, что, согласно данным метода РСА, комплекс 1 имеет структуру изомера A [5]. В то же время $\eta^{2}$-координация на атоме Pt предполагается в комплексах с хелатными фосфиновыми Р-P лигандами при атоме платины $\mathrm{CpReFePt}\left(\mu_{3} \mathrm{C}=\mathrm{CHPh}\right)(\mathrm{CO})_{5}(\mathrm{P}-\mathrm{P})(\mathrm{P}-\mathrm{P}=\mathrm{dppe}$ или dppp) [6] и установлена в соединении $\mathrm{CpMnFePt}\left(\mu_{3} \mathrm{C}=\mathrm{CHPh}\right)(\mathrm{CO})_{6}\left(\mathrm{PPh}_{3}\right)-$ марганцевом аналоге комплекса 1 [7]. Использование комплексов одинакового состава позволяет исключить влияние лигандного окружения при атомах металлов на электронные свойства остова $\operatorname{ReFePt}\left(\mu_{3} \mathrm{C}=\mathrm{CHPh}\right)$.

Для анализа электронной структуры изомеров А и Б был применен метод квантовой теории атомов в молекулах (Quantum Theory Atoms In Molecules - QTAIM), основанный на топологическом анализе распределения электронной плотности [8]. Привлекательной особенностью метода QTAIM является то, что электронная плотность представляет собой наблюдаемую характеристику, что позволяет сопоставить данные QTAIM-анализа с экспериментальными данными [9].

\section{Детали расчетов}

Оптимизация структуры изомеров А и Б была выполнена методом функционала плотности (ФП) с обменно-корреляционным функционалом В3LYP [10, 11], реализованном в про-

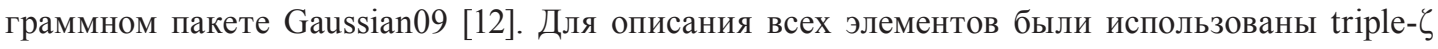
базисные наборы с поляризационными функциями (def2-TZVP), полноэлектронные в случае атомов H, C, O, Р и Fe [13] и псевдопотенциальные для атомов Re и Pt [14]. Все расчеты выполнялись в синглетой электронной конфигурации. Для подтверждения нахождения полученных структур в минимуме потенциальной энергии был проведен расчет нормальных колебаний.

Анализ электронной плотности комплекса 1 был выполнен в рамках QTAIM-метода с помощью программ AIMALL [15] и Multiwfn [16].

\section{Результаты}

Взаимодействия металл-металл. Рассчитанные структуры изомеров А и Б комплекса 1 представлены на рис. 2. Отметим, что хотя изомер Б до сих пор не зафиксирован спектральными методами и не выделен из раствора, он только на 1,8 кДж/моль менее стабилен, чем изо- 


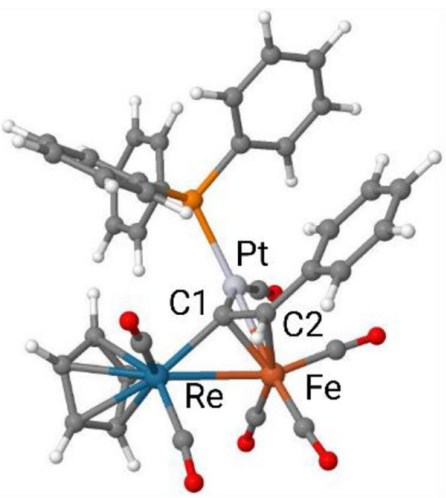

A

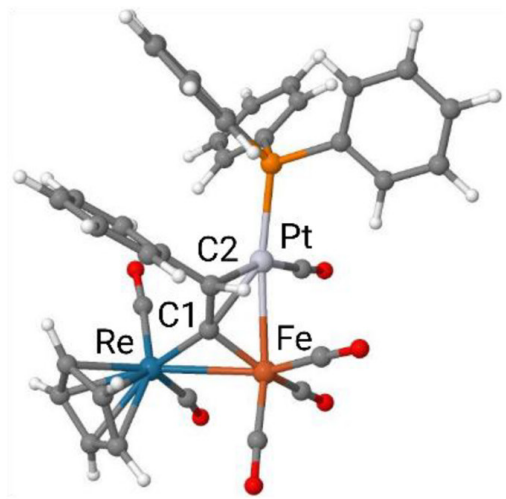

Б

Рис. 2. Рассчитанные структуры изомеров А и Б комплекса 1

Fig. 2. Calculated structures of isomers $A$ and $Б$ of the complex 1

мер А. Таким образом, существование структуры Б с точки зрения квантовой химии является столь же вероятным, как и изомера А. Волновые функции, соответствующие равновесным геометриям обоих изомеров, были использованы для построения электронной плотности.

QTAIM-метод основан на анализе топологических свойств молекулярной электронной плотности $\rho(\mathrm{r})$. Прежде всего, метод пытается локализовать так называемые критические точки $($ КТ), в которых градиент электронной плотности, $\nabla \rho(\mathrm{r})$, равен нулю. Наиболее важными являются точки вблизи атомного ядра (ЯКТ) и связующие точки (СКТ), которые несут информацию о химическом связывании в изучаемой системе. СКТ представляет собой седловую точку второго порядка, которая располагается в минимуме $\rho(\mathrm{r})$ на связывающем пути, соединяющем два атома (две ЯКТ, если быть точнее).

Рисунок 3 демонстрирует молекулярные графы изомеров А и Б комплекса 1, образованных критическими точками и связывающими путями. Прежде всего, мы видим, что QTAIM-анализ показал отсутствие СКТ точек и, как следствие, связывающих путей между всеми тремя атомами металлов, образующих ядро комплекса 1. Так как наличие СКТ между парой атомов является в рамках QTAIM-метода основным индикатором химического связывания [17], можно сделать вывод об отсутствии взаимодействия металл-металл (M-M) в изученных изомерах. Это вполне согласуется с рассчитанными величинами расстояний Re-Pt, paвных 3,47 и 3,81 А в конформерах А и Б соответственно (табл. 1 и 2) [18]. В то же время величины расстояний $\mathrm{Re}-\mathrm{Fe}$ (2,85-2,88 $\AA$ и и $\mathrm{Fe}-\mathrm{Pt}(2,63-2,70 \AA)$ всегда рассматривались как доказательство существования одинарной М-М-связи $[3,5,7]$. Отсутствие связывающего пути между двумя атомами металлов в присутствии мостиково-координированного лиганда является широко распространенным результатом QTAIM-анализа, по крайней мере, в случае предполагаемой одинарной M-Mсвязи [19]. Как следствие, можно говорить о непрямом М-М-взаимодействии, включающем в случае изомеров А и Б карбеновый атом углерода C1 [20-22].

Отсутствие СКТ между Re-, Pt- и Fe-атомами серьезно ограничивает какую-либо интерпретацию М-М-взаимодействия в рамках QTAIM-метода. Альтернативный QTAIM-индикатор химического взаимодействия между атомами, не связанный с присутствием СК точек, - это 


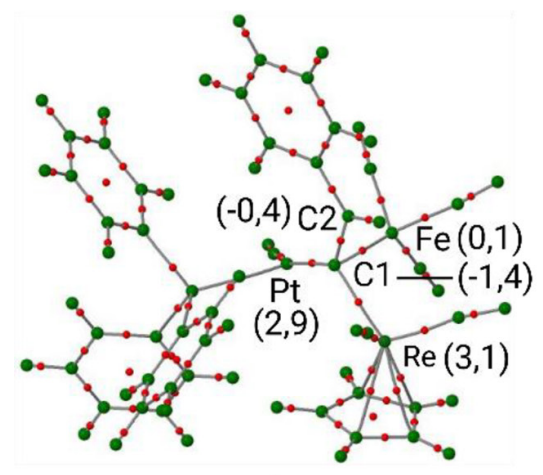

A

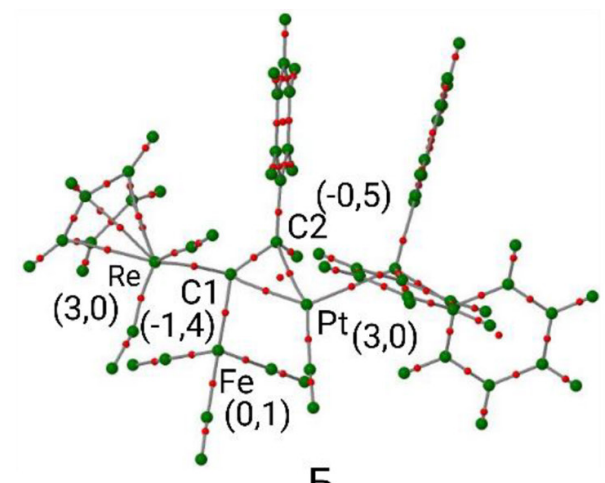

Б

Рис. 3. Молекулярные графы изомеров А и Б, образованные связующими путями и критическими точками. В скобках представлены заряды атомов, рассчитанные QTAIM-методом

Fig. 2. Molecular graphs of isomers A and $\mathrm{B}$, formed by bond paths and critical points. QTAIM atomic charges are shown in parenthesis

индекс делокализации $\delta(\mathrm{A}, \mathrm{B})$. Данная характеристика указывает на число электронных пар, делокализованных между атомами А и В $[23,24]$. В случае связанных атомов $\delta(\mathrm{A}, \mathrm{B})$ используется для оценки степени ковалентности и порядка химической связи [25]. Индексы делокализации, характеризующие М-М-взаимодействия, представлены в табл. 1 и 2. Как и следовало ожидать, величины $\delta(\mathrm{Re}, \mathrm{Pt})$ в обоих комплексах достаточно малы, чтобы свидетельствовать о заметном Re-Pt-взаимодействии, 0,117 (A) и 0,083 (Б). Хотя значения $\delta(\mathrm{Re}, \mathrm{Fe})$ несколько выше, 0,282 (A) и 0,275 (Б), они также указывают на слабое Re-Fe-взаимодействие. Наибольшее M-Mвзаимодействие демонстрируется для пары Fe-Pt. Величины $\delta(\mathrm{Fe}, \mathrm{Pt})$, равные 0,642 (A) и 0,585 (Б), неожиданно высоки в отсутствие связывающего пути и превосходят значения индексов делокализации многих систем с подтвержденной металл-металл-связью [19, 26].

Взаимодействия металл-лиганд. Молекулярные графы указывают на несколько иной способ координации C $=$ CHPh лиганда с ReFePt ядром изомеров А и Б. В случае A QTAIMанализ установил три связывающих пути атома $\mathrm{C} 1$ с каждым из атомов металлов (рис. 3А), что указывает на $\mu_{3}-\eta^{1}: \eta^{1}: \eta^{1}$ способ координации винилидена. Напротив, в изомере Б наблюдаются четыре связывающих пути, которые можно объединить в две группы. Первая группа соответствует взаимодействию $\mathrm{Cl}$ c Re- и Fe-атомами, тогда как вторая характеризует взаимодействие $\mathrm{C} 1=\mathrm{C} 2$ фрагмента с атомом Pt (рис. 3Б). Как следствие, в структуре Б координация винилиденового лиганда может быть описана как $\mu_{3}-\eta^{1}: \eta^{1}: \eta^{2}$ типа. Топологические различия взаимодействия $\mathrm{C} 1=\mathrm{C} 2$ фрагмента с атомом металла в структурах А и Б коррелируют со структурными особенностями изомеров. Если в изомере Б группа C1-Pt-C2 образует симметричный треугольник с расстояниями $\mathrm{Pt}-\mathrm{C} 1$ и $\mathrm{Pt}-\mathrm{C} 2$, равными 2,26 и 2,25 $\AA$ соответственно (табл. 2 ), то в изомере А расстояния $\mathrm{Fe}-\mathrm{C} 1$ и $\mathrm{Fe}-\mathrm{C} 2$ различаются на $0,23 \AA$ (табл. 1).

Пролить свет на природу металл-лигандного взаимодействия помогает ряд характеристик электронной плотности, рассчитываемых в СК-точках (табл. 1 и 2). Во-первых, это величина электронной плотности $\rho(\mathrm{r})$ и знак Лапласиана $\nabla^{2} \rho(\mathrm{r})$. Для всех связывающих путей типа металл-лиганд относительно небольшие значения $\rho(\mathrm{r})$ и положительный знак $\nabla^{2} \rho(\mathrm{r})$ указывают на обеднение электронной плотности в межатомном пространстве и тем самым на ионный ха-

$$
-557-
$$


Таблица 1. Межатомные расстояния $\mathrm{R}(\AA)$, топологические характеристики в СК-точках и индексы делокализации $\delta(\mathrm{A}, \mathrm{B})$ избранных атомных пар изомера А (атомные единицы - au)

Table 1. Interatomic distances $\mathrm{R}(\AA)$, topological indicators of $\rho(\mathrm{r})$ in BCP and delocalization indexes $\delta(\mathrm{A}, \mathrm{B})$ of selected atomic pairs of isomer A (atomic units - au)

\begin{tabular}{c|ccccccccc}
\hline & $\mathrm{R}_{\text {эксп }}{ }^{\mathrm{a}}$ & $\mathrm{R}_{\text {расч }}{ }^{\sigma}$ & $\rho(\mathrm{r})^{\mathrm{B}}$ & $\nabla^{2} \rho(\mathrm{r})^{\mathrm{\Gamma}}$ & $\mathrm{V}(\mathrm{r})^{\text {д }}$ & $\mathrm{G}(\mathrm{r})^{\mathrm{e}}$ & $\mathrm{H}=\mathrm{V}+\mathrm{G}$ & $\mid \mathrm{V} / \mathrm{G}$ & $\delta(\mathrm{A}, \mathrm{B})$ \\
\hline $\mathrm{Re}-\mathrm{C} 1$ & 2,12 & 2,15 & 0,109 & 0,232 & $-0,130$ & 0,090 & $-0,040$ & 1,448 & 0,732 \\
$\mathrm{Fe}-\mathrm{C} 1$ & 1,97 & 1,96 & 0,113 & 0,273 & $-0,168$ & 0,118 & $-0,050$ & 1,422 & 0,639 \\
$\mathrm{Fe}-\mathrm{C} 2$ & 2,20 & 2,24 & & & & & & 0,395 \\
$\mathrm{Pt}-\mathrm{C} 1$ & 2,01 & 2,05 & 0,127 & 0,244 & $-0,175$ & 0,118 & $-0,057$ & 1,485 & 0,893 \\
$\mathrm{Pt}-\mathrm{C} 2$ & 3,05 & 3,07 & & & & & & & 0,089 \\
$\mathrm{Re}-\mathrm{Fe}$ & 2,82 & 2,88 & & & & & & 0,282 \\
$\mathrm{Re}-\mathrm{Pt}$ & 3,35 & 3,47 & & & & & & 0,117 \\
$\mathrm{Fe}-\mathrm{Pt}$ & 2,58 & 2,63 & & & & & & & 0,624 \\
$\mathrm{C} 1-\mathrm{C} 2$ & 1,41 & 1,41 & 0,320 & $-0,789$ & $-0,465$ & 0,134 & $-0,331$ & 3,477 & 1,341 \\
\hline
\end{tabular}

a) Рентгеноструктурные данные; б) расчетные данные; в) электронная плотность; г) лапласиан электронной плотности;

д) плотность потенциальной энергии; 'е) плотность кинетической энергии.

Таблица 2. Рассчитанные межатомные расстояния $\mathrm{R}(\AA)$, топологические характеристики $\rho(\mathrm{r})$ в CKточках и индексы делокализации избранных атомных пар изомера Б (атомные единицы - au)

Table 2. Calculated interatomic distances $\mathrm{R}(\AA)$, topological indicators of $\rho(\mathrm{r})$ in BCP and delocalization indexes $\delta(\mathrm{A}, \mathrm{B})$ of selected atomic pairs of isomer $\mathrm{B}$ (atomic units $-\mathrm{au}$ )

\begin{tabular}{l|cccccccc}
\hline & $\mathrm{R}$ & $\rho(\mathrm{r})^{\mathrm{a}}$ & $\nabla^{2} \rho(\mathrm{r})^{6}$ & $\mathrm{~V}(\mathrm{r})^{\mathrm{B}}$ & $\mathrm{G}(\mathrm{r})^{\mathrm{r}}$ & $\mathrm{H}=\mathrm{V}+\mathrm{G}$ & $\mid \mathrm{V} / \mathrm{G}$ & $\delta(\mathrm{A}, \mathrm{B})$ \\
\hline $\mathrm{Re}-\mathrm{C} 1$ & 2,10 & 0,121 & 0,244 & $-0,151$ & 0,101 & $-0,050$ & 1,489 & 0,776 \\
$\mathrm{Fe}-\mathrm{C} 1$ & 1,87 & 0,146 & 0,391 & $-0,242$ & 0,170 & $-0,072$ & 1,425 & 0,942 \\
$\mathrm{Fe}-\mathrm{C} 2$ & 2,99 & & & & & & 0,058 \\
$\mathrm{Pt}-\mathrm{C} 1$ & 2,26 & 0,080 & 0,162 & $-0,085$ & 0,063 & $-0,022$ & 1,356 & 0,653 \\
$\mathrm{Pt}-\mathrm{C} 2$ & 2,25 & 0,072 & 0,217 & $-0,086$ & 0,070 & $-0,016$ & 1,228 & 0,511 \\
$\mathrm{Re}-\mathrm{Fe}$ & 2,85 & & & & & & & 0,275 \\
$\mathrm{Re}-\mathrm{Pt}$ & 3,81 & & & & & & & 0,083 \\
$\mathrm{Fe}-\mathrm{Pt}$ & 2,70 & & & & & & & \\
$\mathrm{C} 1-\mathrm{C} 2$ & 1,41 & 0,307 & $-0,739$ & $-0,427$ & 0,121 & $-0,306$ & 3,527 & 0,585 \\
\hline
\end{tabular}

а) Электронная плотность; б) лапласиан электронной плотности; в) плотность потенциальной энергии; г) плотность кинетической энергии.

рактер взаимодействия [27]. Однако такая классификация металл-лигандного взаимодействия выглядит слишком упрощенной. Уточнить характер связывания позволяет сумма плотностей кинетической $\mathrm{G}(\mathrm{r})$ и потенциальной $\mathrm{V}(\mathrm{r})$ энергий, $\mathrm{H}(\mathrm{r})=\mathrm{G}(\mathrm{r})+\mathrm{V}(\mathrm{r})$, в СК-точках. Ионный характер взаимодействия проявляется в превалировании энергии $\mathrm{G}(\mathrm{r})$ (всегда положительная величина), что приводит к значениям $\mathrm{H}(\mathrm{r})>0$. Однако в случае изомеров А и Б величина $\mathrm{H}(\mathrm{r})$ меньше нуля для всех металл-винилиден связывающих путей, что указывает на доминирование энергии $\mathrm{V}(\mathrm{r})$ и ковалентный характер взаимодействия $[27,28]$. Можно предположить, что связывание между ReFePt ядром и лигандом $\mathrm{C}=\mathrm{CHPh}$ имеет, по крайней мере, частично ковалентный 
характер. Данный вывод коррелирует и со значениями $\left|\mathrm{V}\left(\mathrm{r}_{\mathrm{b}}\right)\right| / \mathrm{G}\left(\mathrm{r}_{\mathrm{b}}\right)$ параметра, находящимися в интервале от 1 до 2. Это указывает на смешанный характер взаимодействия между парой атомов, тогда как крайние значения $|\mathrm{V}(\mathrm{r})| / \mathrm{G}(\mathrm{r})<1$ и $|\mathrm{V}(\mathrm{r})| / \mathrm{G}(\mathrm{r})>2$ есть индикация «классического» ионного и ковалентного связывания, соответственно [27].

Характеризация металл-лигандного взаимодействия с помощью индексов делокализации показывает высокую степень ковалентности (>0,7), свойственную б-связям [25], в случае $\mathrm{C1}$ карбенового атома, $\eta^{1}: \eta^{1}$ координированного на $\operatorname{RePt}(\mathrm{A})$ и $\operatorname{ReFe}($ Б) парах (табл. 1 и 2). Что касается связи C1-C2 фрагмента с атомом переходного металла, то индексы делокализации указывают на его асимметричность с доминированием C1-M-взаимодействия даже для C1-Pt-C2 группы в изомере Б. Отметим также, что, несмотря на отсутствие в структуре А связывающего пути $\mathrm{Fe}-\mathrm{C} 2$, индекс делокализации $\delta(\mathrm{Fe}, \mathrm{C} 2)$ достаточно высок $(0,395)$, чтобы такое взаимодействие могло быть проигнорировано.

\section{Обсуждение и выводы}

Несмотря на различие в строении изомеров А и Б, связанное с координацией $\mathrm{C}=\mathrm{CHPh}$ лиганда, распределения электронных плотностей и, как следствие, величины зарядов на атомах металлов и винилиденовом лиганде различаются незначительно (рис. 3). Незначительно отличаются и индексы делокализации, характеризующие М-М-взаимодействия в отсутствие связующих путей между металлами. Высокий положительный заряд на атомах Re и Pt в обоих комплексах ( 3,0 е) свидетельствует о смещении электронной плотности в сторону C1 атома, где действительно наблюдается концентрация электронной плотности $(-1,4$ e). В то же время высокие заряды и незначительные величины индексов делокализации указывают на доминирующий электростатический характер взаимодействия между атомами Re и $\mathrm{Pt}$, выражающийся в их взаимном отталкивании и, как результат, большом межатомном расстоянии $(3,47-3,81 \AA)$. Ковалентное взаимодействие $\mathrm{Re}-\mathrm{Fe}$ в обоих комплексах уже достаточно высоко, чтобы его можно было проигнорировать. Как упоминалось выше, величины $\delta(\mathrm{Re}, \mathrm{Fe})$ сравнимы со значениями индексов делокализации в комплексах с М-М-связью, не стабилизированной дополнительно мостиковым лигандом [26]. Re-Fe-расстояния в изомерах А и Б превышают аналогичные дистанции в известных биядерных ReFe-комплексах на 0,17-0,20 А [29]. В то же время это расстояние близко к $\mathrm{Mn}-\mathrm{Fe}-$ расстоянию в $\mathrm{MnFe-аналоге} \mathrm{триметиленметанового} \mathrm{комплекса}$ железа $[3,30,31]$. В отличие от Re-Pt- и Re-Fe-взаимодействий индексы $\delta(\mathrm{Fe}, \mathrm{Pt})$ указывают на сильный ковалентный характер $\mathrm{Fe}-\mathrm{Pt}$-взаимодействия. И это несмотря на отсутствие связующего пути между данными атомами. Более того, индексы делокализации, представленные в табл. 1 и 2 , показывают, что $\delta(\mathrm{Fe}, \mathrm{Pt})$ в обоих комплексах сравнимы с $\delta(\mathrm{Fe}, \mathrm{C} 1)$ и $\delta(\mathrm{Pt}, \mathrm{C} 1)$ в $\pi$-фрагменте C1-M-C2. Данный факт не является чем-то исключительным в QTAIM-анализе. Как показано в ряде работ [32, 33], наличие или отсутствие связующего пути не может считаться однозначным индикатором химической связи и такой параметр, как индекс делокализации, играет более важную роль в характеризации межатомного взаимодействия. Таким образом, можно предположить наличие $\mathrm{Fe}-\mathrm{Pt}$-связи в комплексах А и Б.

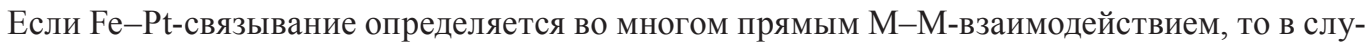
чаe Re-Pt и Re-Fe можно говорить, как упоминалось выше, о непрямом связывании, осуществляемом через мостиковый карбеновый $\mathrm{C} 1$ атом. Взаимодействия Re и $\mathrm{Pt}$ с $\mathrm{C} 1$ в комплексе 
А имеют высокий ковалентный характер, что отражается в распределении лапласиана электронной плотности и свойствах связывающих путей (рис. 4a). В валентной оболочке атома $\mathrm{C1}$ присутствуют два минимума $\nabla^{2} \rho(\mathrm{r})$, соответствующих концентрации электронной плотности (КЗВО), каждый из которых направлен в сторону одного из атомов металлов, т.е. треугольник ReC1Pt стабилизируется двумя двухцентровыми двухэлектронными 2c-2e локализованными взаимодействиями, на что указывает прямой вид Re-C1 и Pt-C1 связующих путей [22]. Похожая картина наблюдается и в комплексе Б (рис. 4в), где лиганд C $=\mathrm{CHPh} \eta^{1}: \eta^{1}$ координируется на ReFe-паре. Однако в последнем случае Re-Cl связующий путь искривлен, свидетельствуя о некоторой делокализации связи металл-углерод [34]. Как результат, мы видим заметное увеличение индекса делокализации $\delta(\mathrm{Re}, \mathrm{Fe})$ в сравнении с $\delta(\mathrm{Re}, \mathrm{Pt})$ в A.

Дополнительным взаимодействием, влияющим на М-М-связывание, является координация атомов $\mathrm{Fe}(\mathrm{A})$ и $\mathrm{Pt}($ Б) на двойной связи $\mathrm{C} 1=\mathrm{C} 2$ винилиденового лиганда. В согласии со структурными особенностями, QTAIM-анализ указывает на сильно асимметричный характер $\mathrm{Fe}-(\mathrm{C} 1=\mathrm{C} 2)$ взаимодействия в изомере $\mathrm{A}$, выражающийся в отсутствии $\mathrm{Fe}-\mathrm{C} 2$ связующего пути и заметно меньшем, в сравнении с $\delta(\mathrm{Fe}, \mathrm{C} 1)$, индексом делокализации $\delta(\mathrm{Fe}, \mathrm{C} 2)$. Отметим, что аналогичная незначительная асимметричность наблюдается и в случае $\mathrm{Pt}-$ $(\mathrm{C} 1=\mathrm{C} 2)$ взаимодействия в структуре Б. Доминирование в той или иной степени $\mathrm{M}-\mathrm{C} 1$ связывания, по-видимому, определяется различием в окружении С1 и С2 атомов, ведущим к концентрации значительной электронной плотности на атоме С1. Связующие пути $\mathrm{M}-\mathrm{C} 1,2$ искривлены в обоих комплексах, что является индикатором донорно-акцепторного взаи-

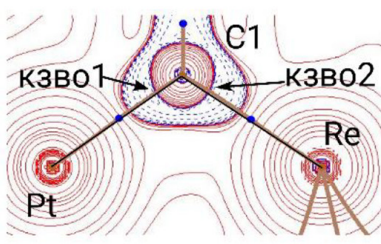

a

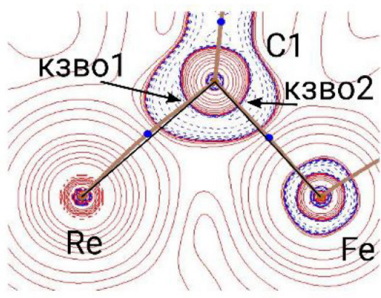

B

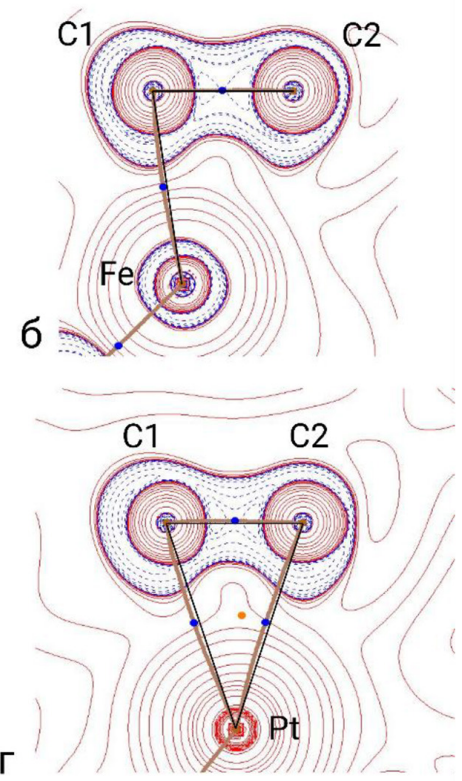

Рис. 4. Проекции лапласиана, $\nabla^{2} \rho(r)$, изомеров А (а, б) и Б (в, г) на плоскости, образованные атомами $\mathrm{ReClPt}(\mathrm{a}), \mathrm{FeClC} 2$ (б), ReC1Fe (в) и PtC1C2 (г). Непрерывные и прерывистые линии соответствуют положительным и отрицательным значениям $\nabla^{2} \rho\left(\mathrm{r}_{\mathrm{b}}\right)$. Показаны также связывающие пути и СК-точки

Fig. 4. The projection of the Laplacian, $\nabla^{2} \rho(r)$, of isomers A (a, б) and Б (в, г) on $\operatorname{ReC} 1 \mathrm{Pt}(\mathrm{a}), \mathrm{FeC} 1 \mathrm{C} 2$ (б), $\mathrm{ReC1Fe}$ (в) и $\mathrm{PtClC} 2$ (г) planes. Positive and negative $\nabla^{2} \rho(\mathrm{r})$ values are marked by solid and dashed lines, respectively. Bond paths and bond critical paints are shown 
модействия [34] металла с алкеновой группой, которое в случае комплекса А смещено в сторону карбенового атома углерода винилиденового лиганда. Несмотря на существенные различия в $\mathrm{M}-(\mathrm{C} 1=\mathrm{C} 2)$ связывании, характер $\mathrm{C} 1-\mathrm{C} 2$-взаимодействия в обоих комплексах практически одинаков. Это проявляется как в длине $\mathrm{C} 1-\mathrm{C} 2$-связи $(1,41 \AA)$, свойственной для комплексов переходных металлов с олефинами $[35,36]$, так и в близких по величине параметрах связывания (табл. 1 и 2), описывающих C1-С2 взаимодействие как ковалентную связь порядка $\sim 1,5$ [37].

Комплекс А можно рассматривать как гетерометаллический аналог триметиленметанового (TMM) комплекса железа [TMM-Fe(CO) 3 , в котором $\mathrm{CH}_{2}$-группы замещены $\mathrm{CpRe}(\mathrm{CO})_{2}$, $\mathrm{Pt}(\mathrm{CO})\left(\mathrm{PPh}_{3}\right)$ и $\mathrm{CHPh}$ фрагментами [38]. Структурные параметры C1-Fe-C2 группы (табл. 1) близки к аналогичным в ТMM- $\mathrm{Fe}(\mathrm{CO})_{3}(\mathrm{C} 1-\mathrm{C} 2=1,43 \AA, \mathrm{Fe}-\mathrm{C} 1=1,95 \AA$ и $\mathrm{Fe}-\mathrm{C} 2=2,14 \AA)$ [32]. Как следствие, топологические характеристики $\mathrm{Fe}-(\mathrm{Cl}=\mathrm{C} 2)$ взаимодействия в обоих комплекcax аналогичны, т.e. QTAIM-анализ комплекса TMM-Fe(CO) $)_{3}$ показывает наличие связующего пути между атомом $\mathrm{Fe}$ и центральным $\mathrm{C} 1$ атомом ТMМ лиганда $(\mathrm{Fe}-\mathrm{C} 1)$ и отсутствие связывания с периферийными атомами С2 [32, 39].

Таким образом, QTAIM-анализ показал, что характер связывания в комплексах А и Б слабо зависит от способа координации винилиденового лиганда. Ни одного металл-металл-связующего пути не было обнаружено в RePtFe-треугольнике, что свидетельствует о непрямом характере взаимодействия между металлами, осуществляемого через $\mu_{3}$-координированный винилиденовый $\mathrm{C}=\mathrm{CHPh}$ лиганд. В то же время индекс делокализации указывает на высокую степень Fe-Pt-взаимодействия, что позволяет предположить существование связи между данными металлами. Это показывает, что связующий путь не всегда является надежным критерием наличия или отсутствия химического взаимодействия. Дополнительное взаимодействие атома металлического ядра с двойной связью $\mathrm{C} 1=\mathrm{C} 2$ винилиденового лиганда в изомере А носит донорно-акцепторный характер и сильно асимметрично, т.е. смещено в сторону атома $\mathrm{C} 1$.

\section{Благодарности / Acknowledgements}

Работа выполнена при финансовой поддержке Российского фонда фундаментальных исследований, Правительства Красноярского края, Красноярского краевого фонда науки в рамках научного проекта «Новые соединения на основе золота и благородных металлов: синтез, физико-химические свойства, каталитическая способность» (грант № 18-43-240010), а также в рамках государственного задания Института химии и химической технологии СО РАН (проект АААА-А17-117021310221-7). Авторы благодарят Сибирский суперкомпьютерный центр СО РАН (г. Новосибирск) за предоставленные вычислительные ресурсы.

The reported study was funded by Russian Foundation for Basic Research, Government of Krasnoyarsk Territory, Krasnoyarsk Regional Fund of Science, in frameworks of the project "New compounds based on gold and noble metals: synthesis, physico-chemical properties, catalytic activity" (grant no. 18-43-240010). This work was conducted within the framework of the budget project AAAA-A17-117021310221-7 for Institute of Chemistry and Chemical Technology SB RAS. The calculations were carried out at the Siberian Supercomputer Center of the Siberian Branch of the Russian Academy of Sciences (Novosibirsk, Russia). 


\section{Список литературы / References}

1. Antonova A.B., Ioganson A.A. Transition metal complexes of unsaturated carbenes: synthesis, structure, and reactivity. Russ. Chem. Rev. 1989. 58 (7), P. 693-710.

2. Bruce M.I. Organometallic chemistry of vinylidene and related unsaturated carbenes. Chem. Rev. 1991. Vol. 919(2), P. 197-257.

3. Antonova A.B. Use of the $\mathrm{Mn}=\mathrm{C}=\mathrm{C}$ system in organometallic and organic synthesis. Coord. Chem. Rev. 2007. Vol. 251 (11-12), P. 1521-1560.

4. Rasmussen S.C. The 18-electron rule and electron counting in transition metalcompounds: theory and application. Chem. Texts 2015. Vol. 1(10).

5. Antonova A.B., Chudin O.S., Vasiliev A.D., Pavlenko N.I.,. Sokolenko W.A, Rubaylo A.I., Semeikin O.V. Chemistry of vinylidene complexes. XVIII. Synthesis and molecular structure of the novel trinuclear $\mu_{3}$-vinylidene complex $\mathrm{CpReFePt}\left(\mu_{3}-\mathrm{C}=\mathrm{CHPh}\right)(\mathrm{CO})_{6}\left(\mathrm{PPh}_{3}\right)$. J. Organomet. Chem. 2009. Vol. 694(1), Р. 127-130.

6. Верпекин В.В., Кондрасенко А.А., Ергаев Р.О., Чудин О.С., Павленко Н.И., Рубайло А.И. Фенилвинилиденовые кластеры с остовом ReFePt, содержащие хелатные дифосфиновые лиганды при атоме платины. Журнал Сибирского федерального университета. Химия. 2017. T. 10(2), C. 239-249 [Verpekin V.V., Kondrasenko A.A., Ergaev R.O., Chudin O.S., Pavlenko N.I., Rubaylo A.I. Phenylvinylidene clusters containing ReFePt metal cores and chelate diphosphine ligands at the platinum atom. Journal of Siberian Federal University. Chemistry. 2017. 10 (2), P. 239-249. (In Russ.)]

7. Chudin O.S., Verpekin V.V., Vasiliev A.D., Rubaylo A.I. Structure of a trinuclear $\mu_{3}-$ vinilydene cluster $\mathrm{CpMnFePt}\left(\mu_{3}-\mathrm{C}=\mathrm{CHPh}\right)\left(\mathrm{PPh}_{3}\right)(\mathrm{CO})_{6}$. J. Struct. Chem. 2017. Vol. 58(3), P. 600-602.

8. Bader R.F.W. A Quantum theory of molecular structure and its applications. Chem. Rev. 1991. Vol. 91(5), P. 893-928.

9. Frenking G., Fröhlich N. The Nature of the bonding in transition-metal compounds. Chem. Rev. 2000. Vol. 100(2), P. 717-774.

10. Becke A.D. Density-functional thermochemistry. III. The role of exact exchange. J. Chem. Phys. 1993. Vol. 98(7), P. 5648-5652.

11. Lee C., Yang W., Parr R.G. Development of the colle-salvetti correlation-energy formula into a functional of the electron density. Phys. Rev. B 1988. Vol. 37(2), P. 785-789.

12. Gaussian 09, Revision D.01, Frisch M.J., Trucks G.W., Schlegel H.B., Scuseria G.E., Robb M.A., Cheeseman J.R., Scalmani G., Barone V., Mennucci B., Petersson G.A., Nakatsuji H., Caricato M., Li X., Hratchian H.P., Izmaylov A.F., Bloino J., Zheng G., Sonnenberg J.L., Hada M., Ehara M., Toyota K., Fukuda R., Hasegawa J., Ishida M., Nakajima T., Honda Y., Kitao O., Nakai H., Vreven T., Montgomery Jr.J.A., Peralta J.E., Ogliaro F., Bearpark M., Heyd J.J., Brothers E., Kudin K.N., Staroverov V.N., Keith T., Kobayashi R., Normand J., Raghavachari K., Rendell A., Burant J.C., Iyengar S.S., Tomasi J., Cossi M., Rega N., Millam J.M., Klene M., Knox J.E., Cross J.B., Bakken V., Adamo C., Jaramillo J., Gomperts R., Stratmann R.E., Yazyev O., Austin A.J., Cammi R., Pomelli C., Ochterski J.W., Martin R.L., Morokuma K., Zakrzewski V.G., Voth G.A., Salvador P., Dannenberg J.J., Dapprich S., Daniels A.D., Farkas O., Foresman J.B., Ortiz J.V., Cioslowski J., Fox D.J. Gaussian, Inc., Wallingford CT, 2013. 
13. Weigend F., Ahlrichs R. Balanced basis sets of split valence, triple zeta valence and quadruple zeta valence quality for H to Rn: design and assessment of accuracy. Phys. Chem. Chem. Phys. 2005. Vol. 7(18), P. 3297-3305.

14. Andrae D., Haeussermann U., Dolg M., Stoll H., Preuss H. Energy-adjusted ab initio pseudopotentials for the second and third row transition elements. Theor. Chim. Acta 1990. Vol. 77(2), P. 123-141.

15. AIMAll (Version 19.02.13), Keith T.A., TK gristmill software, Overland Park KS, USA, 2017 (http: //aim.tkgristmill.com).

16. Lu T., Chen F. Multiwfn: A multifunctional wavefunction analyzer. J. Comput. Chem. 2012. Vol. 3 (5), P. 580-592.

17. Bader R.F.W. A Bond Path: A universal indicator of bonded interactions. J. Phys. Chem. A 1998. Vol. 102(37), P. 7314-7323.

18. Xiao J., Puddephatt R.J. Pt-Re clusters and bimetallic catalysts. Coord. Chem. Rev. 1995. Vol. 143, P. 457-500.

19. Farrugia L.J., Macchi P. Bond orders in metal-metal interactions through electron density analysis. In Electron density and chemical bonding I. Structure and bonding, Springer, Berlin, Heidelberg, 2010. Vol. 146, 127-158 p.

20. Macchi P., Garlaschelli L., Sironi A. Electron density of semi-bridging carbonyls. Metamorphosis of CO ligands observed via experimental and theoretical investigations on $\left[\mathrm{FeCo}(\mathrm{CO})_{8}\right]$. J. Am. Chem. Soc. 2002. Vol. 124(47), P. 14173-14184.

21. Götz K., Kaupp M., Braunschweig H., Stalke D. Comparative analysis of electron-density and electron-localization function for dinuclear manganese complexes with bridging boron and carboncentered ligands. Chem. Eur. J. 2009. Vol. 1 (3), P. 623-632.

22. Farrugia L.J., Evans C. Metal-metal bonding in bridged ligand systems: experimental and theoretical charge densities in $\mathrm{Co}_{3}\left(\mu_{3}-\mathrm{CX}\right)(\mathrm{CO})_{9}(\mathrm{X}=\mathrm{H}, \mathrm{Cl})$, C. R. Chimie 2005. Vol. 8(9-10), P. 1566-1583.

23. Fradera X., Austen M.A., Bader R.F.W. The Lewis model and beyond. J. Phys. Chem. A 1999. Vol. 103(2), P. 304-314.

24. Cortés-Guzmána F., Bader R.F.W. Complementarity of QTAIM and MO theory in the study of bonding in donor-acceptor complexes. Coord. Chem. Rev. 2005. Vol. 249(5-6), P. 633-662.

25. Firme C.L., Antunes O.A.C., Esteves P.M. Relation between bond order and delocalization index of QTAIM. Chem. Phys. Lett. 2009. Vol. 468(4-6), P. 129-133.

26. Macchi P., Sironi A. Chemical bonding in transition metal carbonyl clusters: complementary analysis of theoretical and experimental electron densities. Coord. Chem. Rev. 2003. Vol. 238-239, P. $383-412$.

27. Espinosa E., Alkorta I., Elguero J., Molins E. From weak to strong Interactions: a comprehensive analysis of the topological and energetic properties of the electron density distribution involving X-H $\cdots$ F-Y systems. J. Chem. Phys. 2002. Vol. 117(12), P. 5529-5542.

28. Cremer D., Kraka E., Chemical bonds without bonding electron density-does the difference electron-density analysis suffice for a description of the chemical bond? Angew. Chem., Int. Ed. Engl. 1984. Vol. 23(8), P. 627-628.

29. Zhu B., Yu Y., Chen J., Wu Q., Liu Q. Novel reactions of the rhenium carbyne complex $\left[\left(\eta-\mathrm{C}_{5} \mathrm{H}_{5}\right)(\mathrm{CO})\left(\mathrm{COC}_{2} \mathrm{HB}_{10} \mathrm{H}_{10}\right) \mathrm{ReCC}_{6} \mathrm{H}_{5}\right]$ with metal carbonyl compounds. Crystal structures of 
$\left[\mathrm{ReFe}\left(\mu-\mathrm{CC}_{6} \mathrm{H}_{5}\right)(\mu-\mathrm{CO})(\mathrm{CO})_{3}\left(\eta-\mathrm{C}_{5} \mathrm{H}_{5}\right)\left(\mathrm{COC}_{2} \mathrm{HB}_{10} \mathrm{H}_{10}\right)\right]$ and $\left[\mathrm{ReCo}_{2}\left(\mu_{3}-\mathrm{CC}_{6} \mathrm{H}_{5}\right)(\mu-\mathrm{CO})_{2}(\mathrm{CO})_{5}\left(\eta-\mathrm{C}_{5} \mathrm{H}_{5}\right)\right.$ $\left(\mathrm{C}_{2} \mathrm{HB}_{10} \mathrm{H}_{10}\right)$ ]. Organometal. 1995. Vol. 14(8), P. 3963-3969.

30. Ivanova-Shor E.A., Nasluzov V.A., Shor A.M., Antonova A.B., Rösch N. Vinylidene carbonylation at a manganese-iron complex: A density functional study of mechanism, J. Organomet. Chem. 2001. Vol. 696 (22), P. 3445-3453.

31. Ivanova-Shor E.A., Shor A.M., Nasluzov V.A., Rubailo A.I. A quantum chemical study of the effect of phosphine ligand on the structure of the Mn and Fe vinylidene binuclear complex. J. Struct. Chem. 2016. Vol. 57(2), P. 267-274.

32. Farrugia L.J., Evans C., Tegel M., Chemical bonds without “chemical bonding”? A Combined experimental and theoretical charge density study on an iron trimethylenemethane complex. J. Phys. Chem. A 2006. Vol. 110(25), P. 7952-7961.

33. Shahbazian S. Why bond critical points are not "bond" critical points. Chem. Eur. J. 2018. Vol. 24(21), P. $5401-5405$.

34. Macchi P., Garlaschelli L., Martinengo S., Sironi A. Charge density in transition metal clusters: supported vs unsupported metal-metal interactions. J. Am. Chem. Soc. 1999. Vol. 121 (44), P. $10428-10429$.

35. Nechaev M.S., Rayón V.M., Frenking G. Energy partitioning analysis of the bonding in ethylene and acetylene complexes of group 6, 8, and 11 metals: $(\mathrm{CO})_{5} \mathrm{TM}-\mathrm{C}_{2} \mathrm{H}_{\mathrm{x}}$ and $\mathrm{Cl}_{4} \mathrm{TM}-\mathrm{C}_{2} \mathrm{H}_{\mathrm{x}}$ $(\mathrm{TM}=\mathrm{Cr}, \mathrm{Mo}, \mathrm{W}),(\mathrm{CO})_{4} \mathrm{TM}-\mathrm{C}_{2} \mathrm{H}_{\mathrm{x}}(\mathrm{TM}=\mathrm{Fe}, \mathrm{Ru}, \mathrm{Os})$, and $\mathrm{TM}^{+}-\mathrm{C}_{2} \mathrm{H}_{\mathrm{x}}(\mathrm{TM}=\mathrm{Cu}, \mathrm{Ag}, \mathrm{Au}) . J$. Phys. Chem. A 2004. Vol. 108(15), P. 3134-3142.

36. Scherer W., Eickerling G., Shorokhov D., Gullo E., McGrady G.S., Sirsch P. Valence shell charge concentrations and the Dewar-Chatt-Duncanson bonding model. New J. Chem. 2006. Vol. 30(3), P. 309-312.

37. Bader R.F.W., Tang T.H., Tal Y., Biegler-Koenig F.W. Properties of atoms and bonds in hydrocarbon molecules. J. Am. Chem. Soc. 1982. Vol. 104(4), P. 946-952.

38. Verpekin V.V., Chudin O.S., Piryazev D.A., Rubaylo A.I., Gromilov S.A., Semeikin O.V. New trinuclear $\mu_{3}$-vinylidene cluster $\mathrm{CpReFePt}\left(\mu_{3}-\mathrm{C}=\mathrm{CHPh}\right)(\mathrm{CO})_{5}\left[\mathrm{P}\left(\mathrm{OPr}^{\mathrm{i}}\right)_{3}\right]_{2}$. J. Struct. Chem. 2015. Vol. 56(4), P. 774-776.

39. Mousavi M., Frenking G.J. Bonding analysis of trimethylenemethane (TMM) complexes $\left[(\mathrm{CO})_{3} \mathrm{M}-\mathrm{TMM}\right]\left(\mathrm{M}=\mathrm{Fe}, \mathrm{Ru}, \mathrm{Os}, \mathrm{Rh}^{+}\right)$. Absence of expected bond paths. J. Organomet. Chem. 2013. Vol. 748, P. 2-7. 